\title{
Left atrial dysfunction in patients with obstructive sleep apnea: a combined assessment by speckle tracking and real-time three-dimensional echocardiography
}

\author{
Quan Wan ${ }^{1 \#}$, Guiling Xiang ${ }^{2 \#}$, Yumeng Xing ${ }^{1 \#}$, Shengyu $\mathrm{Hao}^{2}$, Xianhong Shu ${ }^{1}$, Cuizhen Pan ${ }^{1}$, Shanqun $\mathrm{Li}^{2}$ \\ ${ }^{1}$ Department of Echocardiography, Zhongshan Hospital, Fudan University, Shanghai, China; ${ }^{2}$ Department of Respiratory Medicine, Zhongshan \\ Hospital, Fudan University, Shanghai, China \\ Contributions: (I) Conception and design: S Li, C Pan; (II) Administrative support: Q Wan, G Xiang; (III) Provision of study materials or patients: \\ Q Wan, Y Xing; (IV) Collection and assembly of data: Q Wan, G Xiang, S Hao; (V) Data analysis and interpretation: Q Wan, G Xiang, X Shu; (VI) \\ Manuscript writing: All authors; (VII) Final approval of manuscript: All authors. \\ \#These authors contributed equally to this work. \\ Correspondence to: Shanqun Li, MD. Department of Respiratory Medicine, Zhongshan Hospital, Fudan University, 180 Fenglin Road, Shanghai \\ 200032, China. Email: li.shanqun@zs-hospital.sh.cn; Cuizhen Pan. Department of Echocardiography, Zhongshan Hospital, Fudan University, 180 \\ Fenglin Road, Shanghai 200032, China. Email: zhongshanpcz@163.com.
}

Background At present, little research concerning the assessment of left atrial (LA) dysfunction in patients with obstructive sleep apnea (OSA) using a combined assessment by speckle tracking (STE) and real-time three-dimensional echocardiography (RT3DE) is available. The objective of this study was to evaluate the LA volume and function by STE and RT3DE in patients with OSA.

Methods: In our cohort study, ninety-two OSA patients and 50 healthy individuals were enrolled. According to the apnea hypopnea index (AHI), patients (AHI $>15 / \mathrm{h}$ ) classified as having moderate and severe OSA were included. The patients were divided into 2 subgroups according to the left ventricular mass index (LVMI): the left ventricular hypertrophy $(\mathrm{LVH})$ group in which patients had LVH $(\mathrm{n}=30)$, and the nonLVH group in which patients did not have LVH ( $\mathrm{n}=62)$. All subjects underwent LA function assessment by conventional techniques and the combination of STE and RT3DE.

Results: OSA patients showed impaired LA global longitudinal strain during early diastole (LA S-E) and systole (LA S-S) but increased LA global longitudinal strain during late diastole (LA S-A) compared with controls (all $\mathrm{P}<0.05$ ). In addition, OSA patients with LVH had lower LA S-S and LA S-E than patients without LVH (all $\mathrm{P}<0.05$ ). With regard to parameters obtained from RT3DE, indexed LA maximum, minimum, and preatrial contraction volumes (LAVi-max, LAVi-min, LAVi-preA) and the LA active emptying fraction (LAAEF) were significantly higher, whereas the LA passive emptying fraction (LVPEF) was significantly lower in OSA patients in comparison with controls (all $\mathrm{P}<0.05$ ). The LA total emptying fraction (LVTEF) and the LA expansion index were significantly lower in OSA patients with LVH than in controls (all $\mathrm{P}<0.05$ ). Additionally, OSA patients with $\mathrm{LVH}$ had higher LAVi-min, LAVi-preA and LAAEVi but lower LAPEF than patients without LVH (all $\mathrm{P}<0.05)$.

Conclusions: OSA is associated with LA remodeling and dysfunction that occurs in the subclinical stage before the development of $\mathrm{LVH}$ and left ventricular diastolic dysfunction, and it will be further aggravated along with the development of LVH and OSA severity. The process can be detected with a detailed evaluation of active and passive functions of the LA using the STE and RT3DE method.

Keywords: Obstructive sleep apnea (OSA); left atrium; speckle-tracking echocardiography; left ventricular hypertrophy (LVH)

Submitted May 17, 2020. Accepted for publication Nov 25, 2020.

doi: 10.21037/apm-20-1125

View this article at: http://dx.doi.org/10.21037/apm-20-1125 


\section{Introduction}

Obstructive sleep apnea (OSA) is disorder characterized by hypopnea or repetitive apnea as a result of upper airway collapse during sleep (1). As a common chronic illness, OSA impacts an estimated $24 \%$ of men and $9 \%$ of women (2). OSA can cause a variety of diseases and is an independent risk factor for fatal cardiovascular and cerebrovascular events (3-5).

The left atrium (LA) has a crucial role in adjusting left ventricular (LV) filling as follows: receiving blood during ventricular systole, working like a conduit in the early stage of diastole and diastasis, and active contraction as a pump in the late stage of diastole. Several studies have demonstrated chronic effects of OSA on the left ventricle, such as left ventricular hypertrophy $(\mathrm{LVH})$ and $\mathrm{LV}$ diastolic dysfunction (6-9). These conditions were linked with LA remodeling and dysfunction $(10,11)$, which in turn increase the incidence of atrial fibrillation and lead to serious complications during these hypoxia and hypercapnia episodes. Various pathologic factors have been implicated in inducing LA structural and functional remodeling in patients with OSA, including volume overload, endothelial dysfunction, hypoxia, and hypercapnia with increased sympathetic activity and initiation of the inflammatory system $(12,13)$. In OSA patients, apnea hypopnea and an increase in the sympathetic system could induce insulin resistance and renin angiotensin system (RAS) activation, which contribute to myocardial fibrosis.

LA size and function assessed using echocardiography have been demonstrated to detect poor cardiovascular outcomes. Compared with conventional methods, speckle tracking echocardiography (STE) has advantages of semiautomatic character, being less affected by artifacts, and being ultrasonic beam angle-independent. STE is widely used for LA functional assessment in clinical practice (14-16). Two-dimensional STE is a basic technique that does not depend on the angle and analysis function of the longitudinal LA, which has multiple uses, such as the estimation of LV filling pressure and the assessment of LA dyssynchrony. However, myocardial fibers of the LA are arranged in longitudinal and circumferential directions, and we supposed that the evaluation of longitudinal LA function by 2D STE might overlook LA dysfunction. Recently, 3D STE has emerged and developed, and advantages of LA to evaluate volume and phasic function have been reported $(17,18)$. Several echocardiographic studies, including studies of the STE, have shown the relationship between OSA and volume and phasic functions of the LA (19-21). However, the volume calculations may be technically limited by geometric assumptions in biplane Simpson's method. STE and RT3DE were also never assessed together to investigate this issue. Whether a combined method of STE and RT3DE can be used for the assessment of LA function is still unclear.

To address this problem, we first investigated possible subclinical alterations in LA volume and phasic functions by STE and RT3DE in patients with OSA. In the second part of the study, we examined the relationship between echocardiographic parameters of STE and RT3DE and apnea hypopnea index (AHI) in OSA patients. Our aim was to investigate whether LA strain determined by STE and RT3DE is more efficient than conventional methods for identifying OSA patients even in the subclinical stage. Finally, we explored the practicability and repeatability of the method of combining STE and RT3DE for identifying LA remodeling and dysfunction in OSA patients.

We present the following article in accordance with the STROBE reporting checklist (available at http://dx.doi. org/10.21037/apm-20-1125).

\section{Methods}

\section{Study population}

A total of 141 OSA patients who underwent polysomnography (PSG) in the Department of Respiratory Medicine, Fudan University Affiliated Zhongshan Hospital, from April 2015 to September 2018 were enrolled in our study. A total of 50 age- and gender-matched healthy volunteers were recruited and assigned to the control group. Sinus rhythm was normal in all subjects. The criteria for exclusion were hypertension, diabetes mellitus, coronary artery disease, left ventricular systolic dysfunction (LVEF <55\%), congenital heart disease, more than mild valvular heart disease, atrial fibrillation, cardiomyopathy, malignancy, hyperthyroidism, hypothyroidism, and hepatic and renal insufficiency. $\mathrm{LVH}$ is defined as a left ventricular mass index (LVMI) $\geq 150 \mathrm{~g} / \mathrm{m}^{2}$ by echocardiogram. The study was conducted in accordance with the Declaration of Helsinki (as revised in 2013). The study was approved by ethics committee of Fudan University Affiliated Zhongshan Hospital (NO. B2012073R) and informed consent was taken from all the patients. Before echocardiography, we recorded blood pressures, heart rate, $\mathrm{SpO}_{2}$, lipidemia, $\mathrm{FBG}$ and anthropometric measures of all individuals. 


\section{PSG}

Full-night PSG was conducted with an Alice-4 Sleep System (Respironics Inc., Murrysville, PA, USA) with continuous sleep technician monitoring. The PSG assessment incorporated electroencephalography (EEG), electrooculography (EOG) and submental electromyography (EMG). Oxygen saturation was monitored by an oxymeter finger probe. Respiratory movements were monitored by chest and abdominal belts. Airflow was monitored via an oronasal thermistor combined with a nasal pressure sensor. For the registration of the leg movements, tibial anterolateral electrodes were used. A simultaneous electrocardiography (ECG) was recorded. The definition of apnea was cessation of airflow lasting $\geq 10 \mathrm{~s}$. The definition of hypopnea was $\geq 50 \%$ reduction of airflow lasting $\geq 10 \mathrm{~s}$ associated with arousal or $\geq 3 \%$ reduction in arterial oxygen saturation compared to the baseline level. We recorded the total number of episodes of apnea and hypopnea per hour of sleep to calculated the AHI. The diagnosis of OSA was made if only the $\mathrm{AHI}$ index was more than 5, and then patients were classified as mild (AHI 5-14), moderate (AHI 15-30) and severe (AHI >30) (22).

\section{Transthoracic echocardiography}

One experienced cardiologist who was blinded to the grouping information performed transthoracic echocardiography using an iE33 ultrasound system (Philips Medical System, Andover, MA, USA) and an S5-1 transducer. The position of the patient was left lateral decubitus. We measured several indexes following international guidelines, including LV end-diastolic (LVEDD) and end-systolic (LVESD) diameter, diastolic interventricular septum, LV posterior wall thickness, LA diameter and LV mass (23). We chose the biplane Simpson's method to obtain the LVEF. Pulsed-wave Doppler echocardiography was used to obtain the E-wave deceleration time (DT) and the $\mathrm{E}$ and A values through the mitral valves. We also used tissue Doppler imaging to obtain the E' and S' values of the mitral annulus and subsequently calculated the E/E' and E/A ratios. All echocardiographic images were saved in cineloop format.

\section{STE}

For STE analysis, we adopted a high frame rate ranging from 50 to 80 frames/sec to reliably record images from apical four- and two-chamber views. Three consecutive cardiac cycles were stored in digital format, and then we analyzed them using a software system (Philips QLAB10.4 software). The software could automatically calculate the endocardial border traced manually and epicardially. We adjusted the region of interest (ROI) manually, and then software automatically divided the LA myocardial wall into six segments. Segments with inadequate tracking quality were excluded from the analysis. During systole (LA S-S), the LA strain was obtained just before mitral valve opening and at the beginning of the $\mathrm{P}$ wave on the ECG strain during late diastole (LA S-A). During early diastole, the LA strain (LAS-E) was obtained by subtracting LA S-A from LA S-S. The final LA strain values were obtained from the average values of each apical view.

\section{Real-time 3D echocardiography}

RT3DE was conducted by an iE33 ultrasound system (Philips Medical Systems) and an X5-1 matrix-array transducer (1-3 MHz). In a "full-volume" set, all RT3DE images were obtained from four apical chamber views. We recorded at least four consecutive cardiac cycles. Five points were marked on the atrial surface of the mitral annulus (anterior, inferior, lateral, and septal). The fifth point was at the apex of the LA. Software could automatically detect each frame of the LA internal endocardial border. Therefore, we generated a 3D model of the LA volume (Figure 1). The indexes measured were as follows: maximum LA volume at end systole (LAVmax), minimum LA volume at end diastole (LAVmin), and LA volume before atrial contraction (LAVpreA) (Figure 2). According to previous studies $(24,25)$, the following indexes were obtained: (I) LA reservoir function: LA total emptying volume $=\mathrm{LAVmax}-$ LAVmin, LA total emptying fraction $(\mathrm{LVTEF})=(\mathrm{LAV} \max$ $-\mathrm{LAVmin}) / \mathrm{LAV} \max \times 100, \mathrm{LA}$ expansion index $=(\mathrm{LAVmax}$ - LAVmin)/LAVmin $\times 100$; (II) LA conduit function: LA passive emptying volume $=\mathrm{LAV} \max -\mathrm{LAVpreA}, \mathrm{LA}$ passive emptying fraction $(\mathrm{LVPEF})=(\mathrm{LAVmax}-\mathrm{LAV}$ preA $) /$ LAVmax $\times 100$; and (III) LA booster pump function: LA active emptying volume $=\mathrm{LAVpreA}-\mathrm{LAVmin}, \mathrm{LA}$ active emptying fraction $(\mathrm{LAAEF})=(\mathrm{LAV}$ preA-LAVmin $) /$ LAVpreA $\times 100$. Two experienced observers who were blinded to grouping information analyzed all digital data.

\section{Reproducibility}

We selected twenty patients randomly to evaluate the 

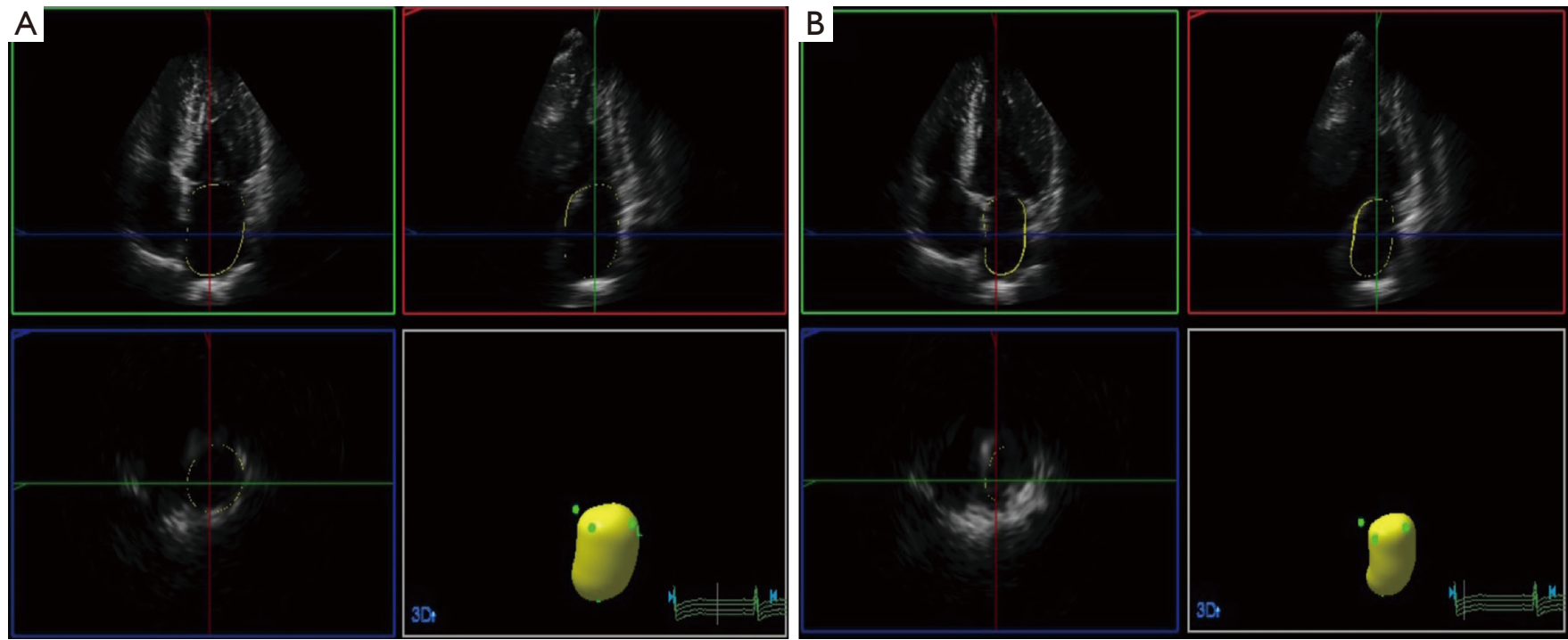

Figure 1 Real time three-dimensional echocardiography of maximal left atrial volume (A) and minimal left atrial volume (B).

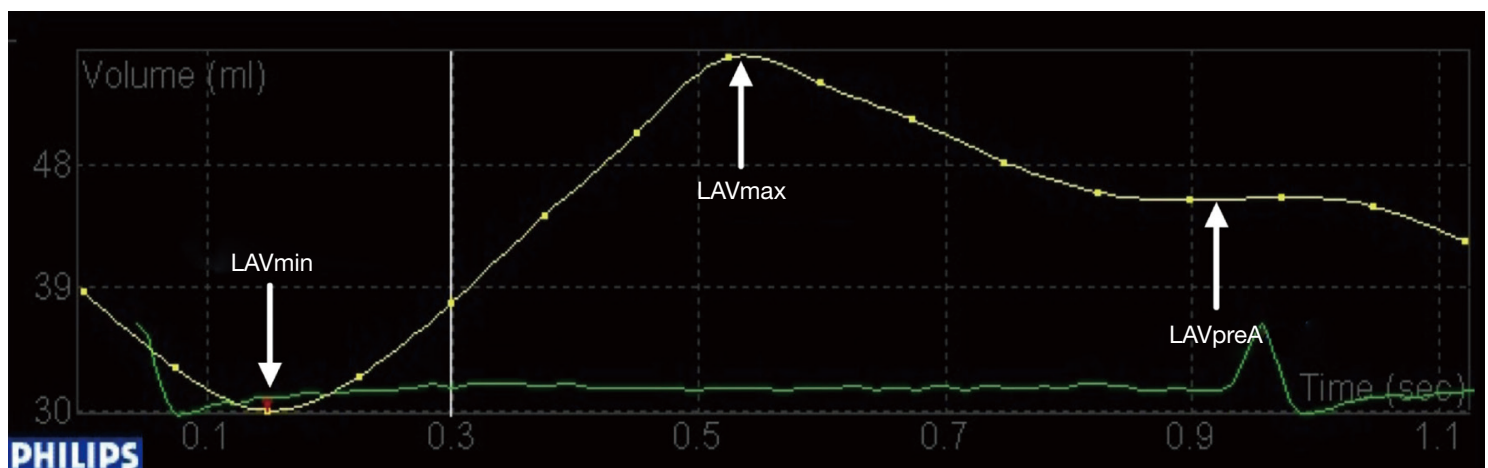

Figure 2 Time-volume curve showing the dynamic change of left atrial maximal volume (LAVmax), left atrial minimal volume (LAVmin), and before left atrial contraction volume (LAVpreA).

intra- and interobserver variability. To avoid recall bias, one observer measured speckle tracking and RT3DE data twice, and then intraobserver variability was obtained. A second observer who was blinded to the result from the first observer assessed the interobserver variability independently.

\section{Statistical analysis}

All statistical analyses were conducted with SPSS 21.0 software (SPSS Inc., Chicago, IL, USA). The Kolmogorov-Smirnov test was used to analyze the distribution of variables. Continuous variables are shown as the mean \pm standard deviation $(\mathrm{SD})$ or median (interquartile range) appropriately. The categorical variables are shown as percentages. For normally distributed variables, one-way ANOVA was used for comparisons between two groups. For variables with abnormal distributions, the Kruskal-Wallis test was used for comparisons between two groups. Post hoc comparisons were performed with the Scheffé test. The relationships between LA strain and RT3DE volumes and clinical indexes were measured by the Pearson correlation test. We used the intraclass correlation coefficient (ICC) and Bland-Altman analysis to measure the inter- and intraobserver reproducibility of speckle tracking and RT3DE data. A P value less than 0.05 was considered statistically significant. 
Table 1 Baseline characteristics and conventional echocardiography

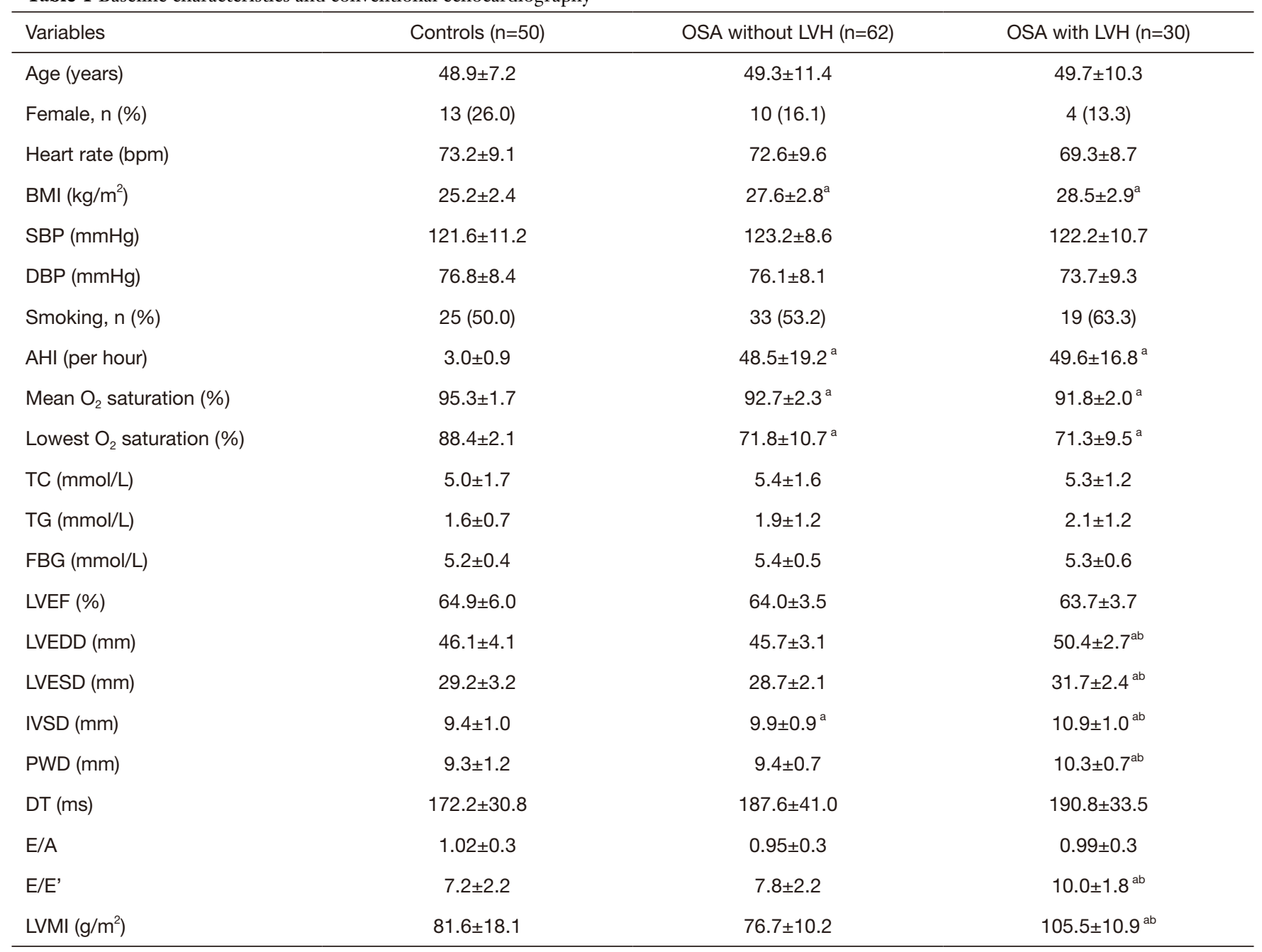

a, $\mathrm{P}<0.05$, compare between OSA group and controls; ${ }^{b}, \mathrm{P}<0.05$, compare between OSA with LVH group and without LVH group. OSA, obstructive sleep apnea; LVH, left ventricular hypertrophy; BMI, body mass index; SBP, systolic blood pressure; DBP, diastolic blood pressure; AHI, apnea hypopnea index; TC, total cholesterol; TG, triglycerides; FBG, fasting blood glucose; LVEF, left ventricular ejection fraction; LVEDD, left ventricular end-diastolic diameter; LVESD, left ventricular end-systolic diameter; IVSD, interventricular septum diastolic thickness diameter; PWD, posterior wall diastolic thickness diameter; DT: deceleration time; E/A ratio between early and late diastolic inflow velocities; E/E', ratio between early diastolic inflow velocity and early diastolic annular myocardial velocity; LVMI, left ventricular mass index.

\section{Results}

\section{Study population}

Among the 141 enrolled OSA patients, 42 patients with mild OSA (AHI between 5 and 14) and 7 patients with suboptimal tracking quality were excluded. Ninety-two patients with moderate to severe OSA (non-LVH group: 10 women and 52 men, mean age, $49.3 \pm 11.4$ years; $\mathrm{LVH}$ group: 4 women and 26 men, mean age, $49.7 \pm 10.3$ years) and 50 healthy controls (13 women and 37 men; mean age, $48.9 \pm 7.2$ years) were ultimately enrolled.

\section{Clinical characteristics and conventional echocardiography}

The clinical characteristics and conventional echocardiography results are shown in Table 1. BMI and AHI were higher in the OSA groups than in the control group. However, compared with the control group, the mean $\mathrm{SpO}_{2}$ and the lowest $\mathrm{SpO} 2$ in the OSA groups were lower (all $\mathrm{P}<0.05$ ). For conventional echocardiographic data, OSA patients with LVH showed significantly higher LVEDD, LVESD, IVSD, PWD, E/E' and LVMI than OSA patients without $\mathrm{LVH}$ and controls $($ all $\mathrm{P}<0.05)$. Echocardiographic 
Table 2 Speckle-tracking and RT3DE parameter

\begin{tabular}{|c|c|c|c|}
\hline Variables & Controls $(n=50)$ & OSA without LVH $(n=62)$ & OSA with LVH $(n=30)$ \\
\hline LA S-E (\%) & $23.9 \pm 3.8$ & $15.1 \pm 3.6^{a}$ & $12.2 \pm 3.3^{\mathrm{ab}}$ \\
\hline LA S-A (\%) & $15.3 \pm 2.8$ & $18.9 \pm 5.3^{a}$ & $17.9 \pm 4.1^{\mathrm{a}}$ \\
\hline LAVi-max $\left(\mathrm{mL} / \mathrm{m}^{2}\right)$ & $21.9 \pm 3.4$ & $24.5 \pm 5.1^{\mathrm{a}}$ & $26.6 \pm 5.7^{a}$ \\
\hline LAVi-min $\left(\mathrm{mL} / \mathrm{m}^{2}\right)$ & $8.3 \pm 1.8$ & $9.7 \pm 2.3^{a}$ & $10.8 \pm 2.3^{\mathrm{ab}}$ \\
\hline LATEVi $\left(\mathrm{mL} / \mathrm{m}^{2}\right)$ & $13.6 \pm 2.2$ & $14.8 \pm 3.5$ & $15.7 \pm 3.8^{a}$ \\
\hline LATEF (\%) & $62.3 \pm 4.8$ & $60.3 \pm 5.8$ & $59.0 \pm 4.0^{\mathrm{a}}$ \\
\hline LAAEVi $\left(\mathrm{mL} / \mathrm{m}^{2}\right)$ & $5.9 \pm 1.4$ & $9.0 \pm 2.7^{\mathrm{a}}$ & $10.2 \pm 2.9^{\mathrm{ab}}$ \\
\hline LAPEF (\%) & $35.4 \pm 6.6$ & $23.8 \pm 5.3^{a}$ & $20.8 \pm 3.9^{\mathrm{ab}}$ \\
\hline LA expansion index (\%) & $169.1 \pm 34.5$ & $157.5 \pm 39.7$ & $146.3 \pm 25.2^{\mathrm{a}}$ \\
\hline
\end{tabular}

${ }^{a}, \mathrm{P}<0.05$, compare between OSA group and controls; ${ }^{\mathrm{b}}, \mathrm{P}<0.05$, compare between OSA with LVH group and without LVH group. OSA obstructive sleep apnea; left ventricular hypertrophy (LVH); LA S-S, left atrial global longitudinal strain during systole; LA S-E, left atrial global longitudinal strain during early diastole; LA S-A, left atrial global longitudinal strain during late diastole; LAVi-max, indexed left atrial maximum volume; LAVi-preA, indexed left atrial pre-atrial contraction volume; LAVi-min, indexed left atrial minimum volume; LATEVi, indexed left atrial total emptying volume; LATEF, left atrial total emptying fraction; LAAEVi, indexed left atrial active emptying volume; LAAEF, left atrial active emptying fraction; LAPEVi, indexed left atrial passive emptying volume; LAPEF, left atrial passive emptying fraction.

parameters other than IVSD were not different between the non-LVH group and the control group (all P>0.05).

\section{Speckle-tracking and RT3DE data}

As shown in Table 2 and Figure 3, the LA S-S and LA S-E were significantly lower and LA S-A was higher in the OSA group than in the controls (all $\mathrm{P}<0.05$ ). Notably, LA S-S and LA S-E values in the OSA with LVH group were lower than those in the non-LVH group (all $\mathrm{P}<0.05$ ). For the analysis of RT3DE parameters, the LAVi-max and LAAEF values were significantly higher, while LAPEVi was significantly lower in both OSA groups, especially in the LVH group, compared to the control group (all $\mathrm{P}<0.05$ ). The LAVi-preA, LAVi-min, and LAAEVi values in the LVH group were higher, but the LAPEF value was lower, in comparison with the non-LVH group and the control group (all $\mathrm{P}<0.05$ ). No difference was found between the nonLVH group and the control group regarding the LATEVi, LATEF and LA expansion index $(\mathrm{P}>0.05)$. The LATEVi, LATEF and LA expansion index in the LVH group were lower than those in the control group (all $\mathrm{P}<0.05$ ).

\section{Relationship between echocardiographic parameters and AHI}

Positive correlations were observed between AHI and LAVi-max, LAVi-min, LAVi-preA, LATEVi, and LAAEVi values. Meanwhile, the correlations between AHI and LA $\mathrm{S}-\mathrm{S}$ and LA S-E were inverse (all $\mathrm{P}<0.05$ ). No correlations were found between AHI and LA S-A, LATEF, LAAEF, LAPEVi, LAPEF, and LA expansion index (all P>0.05, Table 3).

\section{Reproducibility}

We randomly selected twenty OSA patients to evaluate the reproducibility of speckle tracking and RT3DE. The ICCs of LA S-S, LA S-E, LA S-S, LA-max, LA-pre, and LA-min were 0.958 for intraobserver variability $(\mathrm{P}<0.001,95 \% \mathrm{CI}$ : 0.897-0.983), 0.966 ( $\mathrm{P}<0.001,95 \%$ CI: 0.917-0.987), 0.927 $(\mathrm{P}<0.001,95 \%$ CI: 0.825-0.970), $0.940(\mathrm{P}<0.001,95 \%$ CI: 0.856-0.976), 0.909 (P<0.001, 95\% CI: 0.785-0.963) and $0.883(\mathrm{P}<0.001,95 \% \mathrm{CI}: 0.728-0.952)$, respectively. The ICCs of LA S-S, LA S-E, LA S-S, LA-max, LA- 

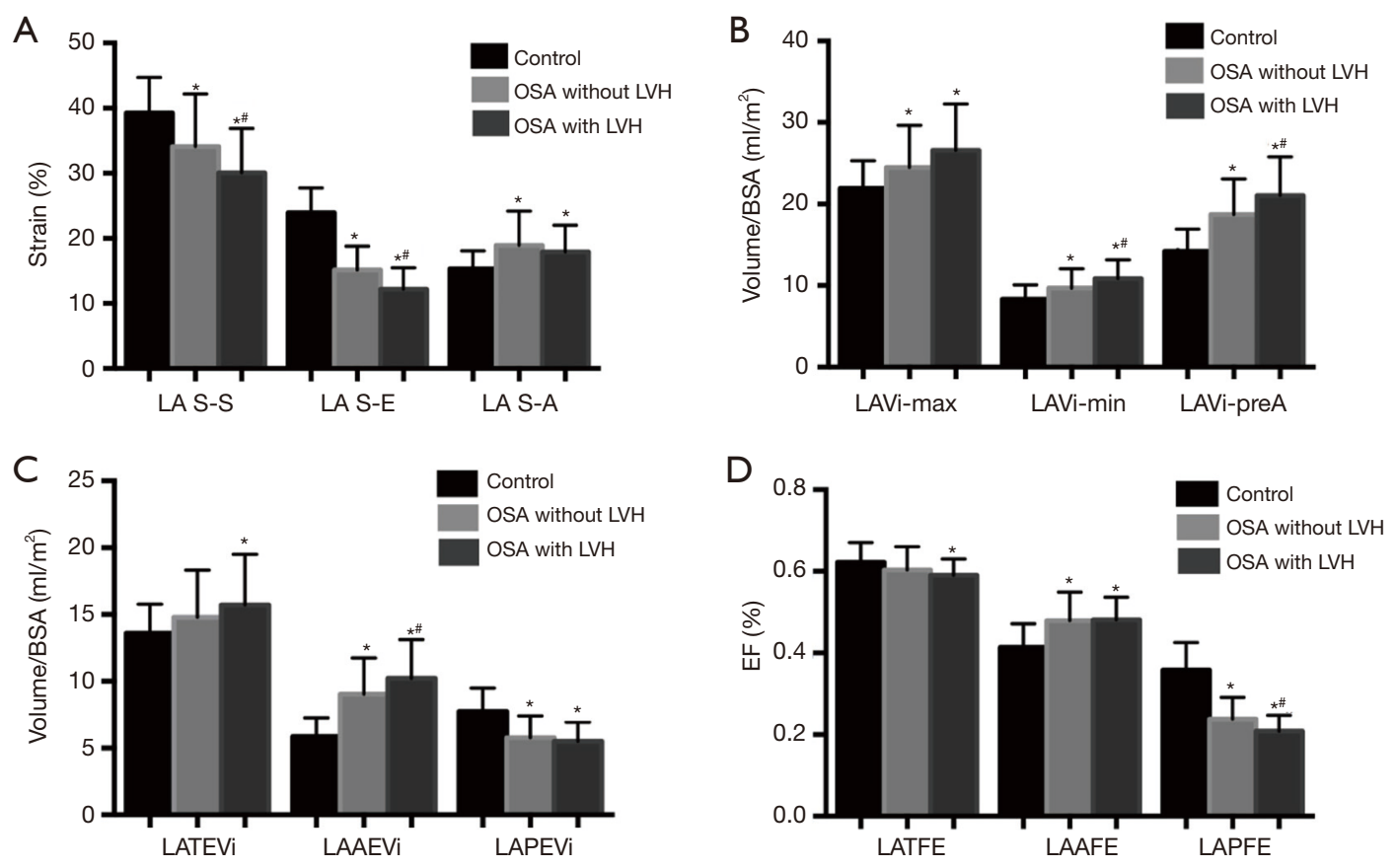

Figure 3 Speckle-tracking and real-time three-dimensional echocardiography (RT3DE) measure. *, $\mathrm{P}<0.05$, compare between obstructive sleep apnea (OSA) group and controls;,, $\mathrm{P}<0.05$, compare between OSA with left ventricular hypertrophy (LVH) group and without LVH group.

pre, and LA-min were 0.915 for interobserver variability $(\mathrm{P}<0.001,95 \%$ CI: 0.797-0.965), $0.893(\mathrm{P}<0.001,95 \%$ CI: 0.750-0.956), 0.893 ( $\mathrm{P}<0.001,95 \%$ CI: 0.730-0.958), 0.902 $(\mathrm{P}<0.001,95 \%$ CI: 0.770-0.960), $0.869(\mathrm{P}<0.001,95 \%$ CI: 0.699-0.946) and $0.867(\mathrm{P}<0.001,95 \%$ CI: 0.694-0.945), respectively. The results showed satisfactory reproducibility of the strain measurement (Figure 4).

\section{Discussion}

Various factors, especially hypoxia and hypercapnia, which ensue during sleeping, were reported to have negative effects on structural and functional cardiac remodeling in OSA patients, which may cause increased cardiovascular morbidity and mortality (26). Frequent apneas expose the cardiovascular system to increased negative intrathoracic pressure, leading to the activation of sympathetic activity and hemodynamic changes. Chronic damage to myocardial cells could lead to apoptosis development, and myocardial fibrosis may accelerate cardiac dysfunction development. Intermittent hypoxia associated with OSA significantly influences the cardiovascular system and causes myocardial damage through actions on the sympathetic nervous system and endothelial function $(12,13)$.

Our study is the first to quantitatively evaluate LA phasic functions by combining STE and RT3DE in patients with moderate to severe OSA. Our results show that the reservoir and conduit functions assessed by STE and RT3DE were impaired in OSA patients with or without $\mathrm{LVH}$, while active pump function was enhanced. In addition, the AHI determines the severity of LA dysfunction in OSA patients. As the most extensively used tool to assess cardiac function, conventional echocardiography is suitable for clinical practice. Nevertheless, there are several limitations related to the geometry and shape of LA and LA-focused views needed. The application of conventional echocardiography in everyday clinical practice is not sensitive enough for the earlier detection of subtle myocardial dysfunction. STE has advantages over semiautomatic characterization and the lack of geometric assumptions, as it is angleindependent and provides frame-by-frame tracking of natural acoustic markers. Moreover, STE is less sensitive to signal noise and is not impacted by translational movement, such as respiration or tethering, caused by the adjacent myocardium. STE has been gradually used for LA functional assessment in clinical practice to upgrade the role 
Table 3 Correlations of STE and RT3DE parameters and AHI

\begin{tabular}{lcc}
\hline & \multicolumn{2}{c}{ AHI } \\
\cline { 2 - 3 } & $r$ & $P$ \\
\hline LA S-S (\%) & -0.442 & 0.024 \\
LA S-E (\%) & -0.421 & 0.032 \\
LA S-A (\%) & -0.314 & 0.118 \\
LAVi-max $\left(\mathrm{mL} / \mathrm{m}^{2}\right)$ & 0.531 & 0.005 \\
LAVi-preA $\left(\mathrm{mL} / \mathrm{m}^{2}\right)$ & 0.548 & 0.004 \\
LAVi-min $\left(\mathrm{mL} / \mathrm{m}^{2}\right)$ & 0.416 & 0.035 \\
LATEVi $\left(\mathrm{mL} / \mathrm{m}^{2}\right)$ & 0.546 & 0.004 \\
LATEF $(\%)$ & 0.186 & 0.362 \\
LAAEVi $\left(\mathrm{mL} / \mathrm{m}^{2}\right)$ & 0.563 & 0.003 \\
LAAEF $(\%)$ & 0.275 & 0.174 \\
LAPEVi $\left(\mathrm{mL} / \mathrm{m}^{2}\right)$ & 0.294 & 0.145 \\
LAPEF $(\%)$ & -0.172 & 0.402 \\
LA expansion index (\%) & 0.204 & 0.317 \\
\hline AHI apnea $\mathrm{hypOpnea}$ & &
\end{tabular}

AHI, apnea hypopnea index; LA S-S, left atrial global longitudinal strain during systole; LA S-E, left atrial global longitudinal strain during early diastole; LA S-A, left atrial global longitudinal strain during late diastole; LAVi-max, indexed left atrial maximum volume; LAVi-preA, indexed left atrial pre-atrial contraction volume; LAVi-min, indexed left atrial minimum volume; LATEVi, indexed left atrial total emptying volume; LATEF, left atrial total emptying fraction; LAAEVi, indexed left atrial active emptying volume; LAAEF, left atrial active emptying fraction; LAPEVi, indexed left atrial passive emptying volume; LAPEF, left atrial passive emptying fraction.

of echocardiography (14-16). Furthermore, RT3DE may overcome these abovementioned limitations, as an advanced technique allows comprehensive, reproducible, quantitative, volumetric evaluation of the LA volume and phasic function and no longer depends on geometric assumptions with better spatial and temporal resolution $(17,18)$. Theoretically, combining STE and RT3DE to evaluate LA dysfunction is superior to conventional echocardiography, such as DTI or 2D STE.

In previous studies, LA volume and dysfunction were increased in OSA patients, along with LVH and diastolic dysfunction. LA failure may be partially caused by elevated LV filling pressures. Fibrosis in the left atrium, which plays a key role in systolic and diastolic dysfunction of the left atrium, could disrupt myocardial functions $(19,27)$. In OSA patients without LVH, RT3DE could detect
LA longitudinal dysfunction, even without morphologic changes in the LA, although conventional echocardiography could not detect LA dysfunction. The severity of LA fibrosis and the relationship between the LASE and LASE values verify the correlation between the involved parameters and LA fibrosis.

In the early phases of OSA, left ventricle diastolic dysfunction and increased left ventricle filling pressure develop and then lead to LA passive emptying decreases. In our research, we confirmed that conduit functions of the LA (LASE, LAPEVi, LAPEF), reservoir function of the LA (LASS, LVTEF) and left ventricle filling decreased, and the pump function of the LA (LASA, LVAEF) increased in both OSA groups, especially in the LVH group, in comparison with the control group. Movements of myocardial tissue tend to slow down, and passive emptying of the LA is delayed, despite passive deformation of the LA myocardium. LA remodeling combined with myocardial damage and fibrosis may negatively impact the movements of the LA, resulting in LA dysfunction in the OSA group. The conduit and reservoir functions of the LA in the LVH group were lower than those in the non-LVH group. The results indicate that active discharge of the LA would be more easily caused by LA myocardial fibers with more common contractions in the non-LVH group. Thus, in the subclinical phases of OSA, passive emptying function of the LA is retained. Positive correlations were observed between AHI and LAVi-max, LAVi-min, LAVi-preA, LATEVi, and LAAEVi values. Meanwhile, inverse relationships were detected between AHI and LA SS and LA SE. Therefore, we suppose that LA volume and functional changes occur earlier than morphological changes of LV in OSA patients.

The major mechanism of OSA is hypertrophy of left ventricular and diastolic dysfunction. On the one hand, repeated hypoxia causes renin-angiotensin aldosterone system activation, directly leading to myocardial fibrosis and thinning of the wall; on the other hand, hypoxia causes sympathetic activation and enhances peripheral vascular resistance, and increased postload affects $L V$ systolic function and results in reduced wall compliance. To maintain adequate LV filling, LVH will deteriorate. The increase in LV filling pressure shown by E/E' values causes LA overstretch and an increase in chronic LA afterload. Changes in the structure of the LA may influence conduit and reservoir functions in OSA patients with LVH. The reservoir function of the LA, which is related to protecting the relaxation function, is compensated by increasing the LA volume and the wall tension. In the early stage of OSA, 
LA S-S

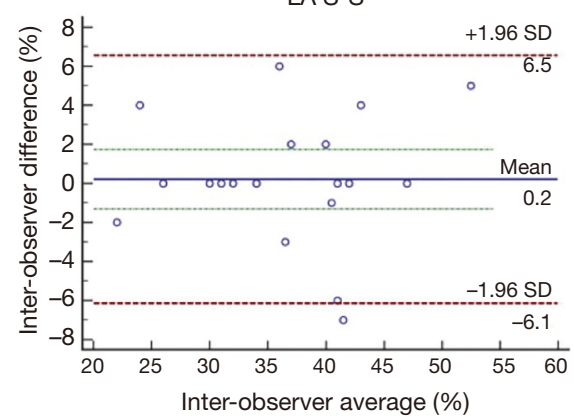

LAVi-max

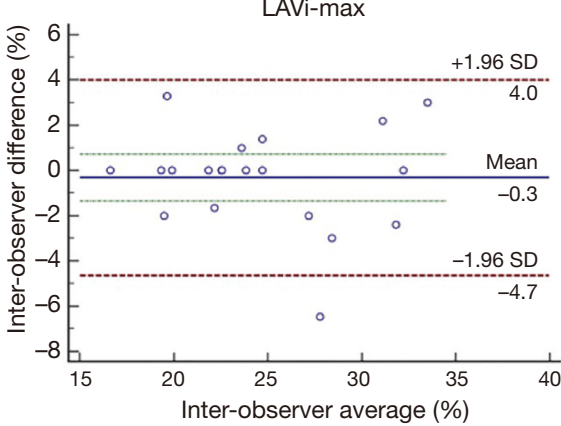

LA S-S

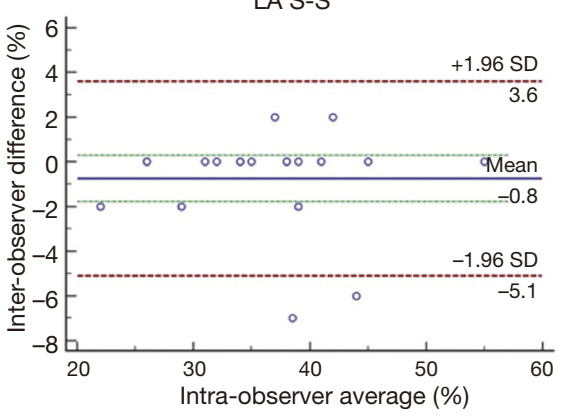

LAVi-max

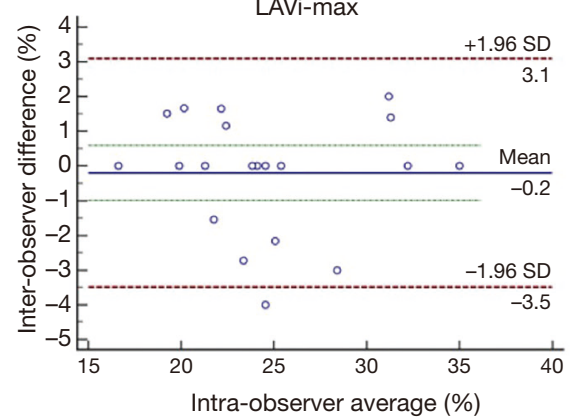

LA S-E

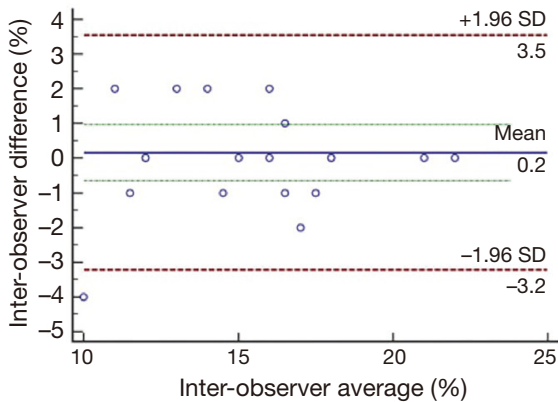

LAVi-min

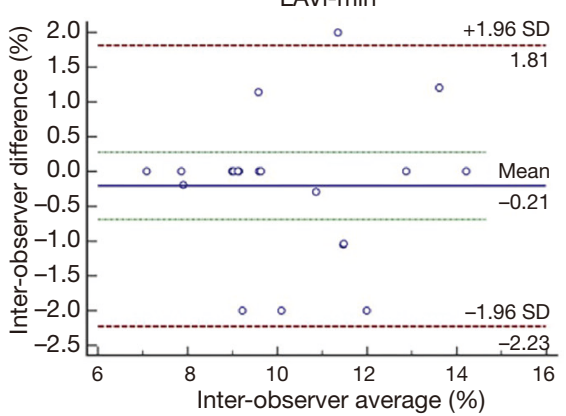

LA S-E

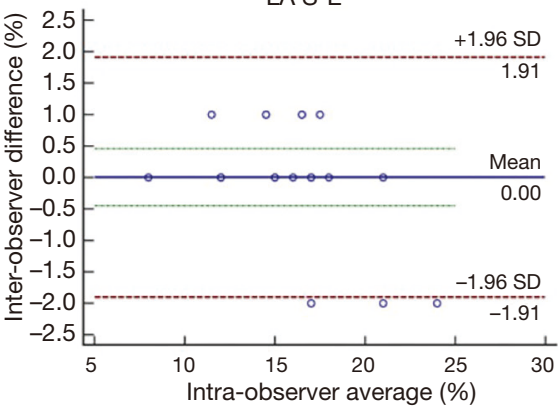

LAVi-min

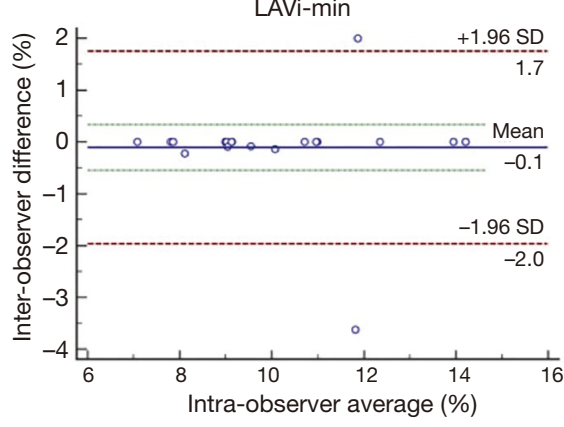

LA S-A

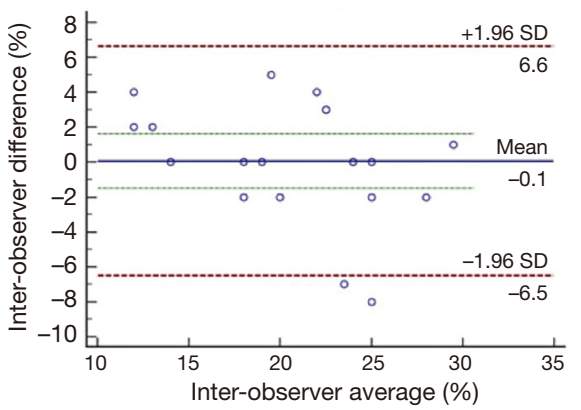

LAVi-preA

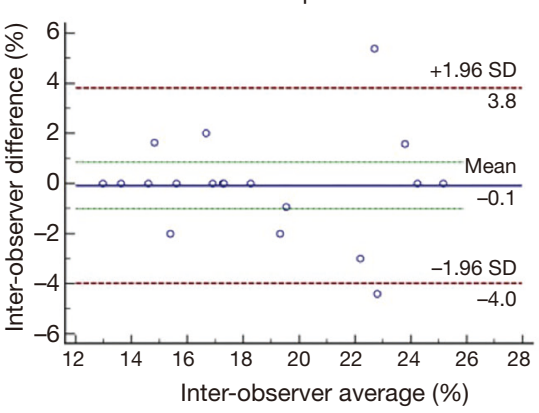

LA S-A

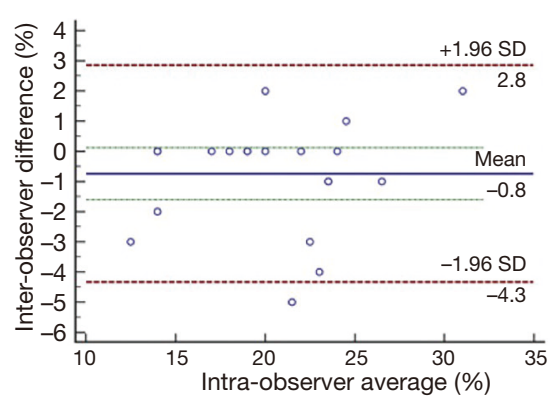

LAVi-preA

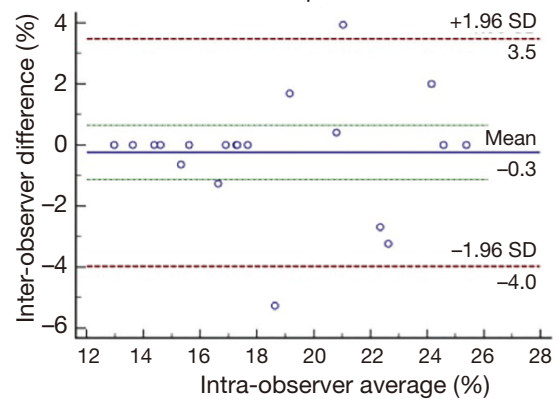

Figure 4 Bland-Altman plots showing differences between interobserver and intraobserver and limits of agreement of real-time threedimensional echocardiography (RT3DE) parameters.

the conduit function of the LA deteriorates. The pump function is activated to compensate for the conduit and reservoir function. Additionally, left ventricle filling reaches a fairly high level (28). Notably, our results indicated satisfactory reproducibility of the strain measurement, which could be explained by $3 \mathrm{D}$ software with higher degree of automation. Taken together, we found that the STE and RT3DE methods could detect LA dysfunction 
in OSA patients even without LVH. Hence, the STE and RT3DE methods help to distinguish patients with normal $\mathrm{LV}$ sizes that have an increased risk for LVH from those with normal LV sizes that have a low risk for LVH. That is, combined STE and RT3DE may be the better method for identifying OSA patients, even in the subclinical phase.

There were several limitations in our study. First, the sample size in the study was relatively small. Further longterm follow-up studies with large samples are urgently needed to assess the diagnostic value of LA volumes and mechanical functions for OSA patients. Second, LA appendage plays a key role in LA reservoir function, but we did not include it in the calculations of LA volume and function. Third, we did not evaluate LA volume by MRI or computerized tomography other than RT3DE. Fourth, our study lacked a control group of obese patients with similar BMIs but without OSA.

\section{Conclusions}

Our study showed LA volume and phasic functions were deteriorated in all OSA patients with LVH or without LVH. STE and RT3DE may contribute to recognition of subclinical myocardial alternations which were secondary to OSA by measuring atrial volume and function. Further prospective studies with large sample are needed to evaluate the prognostic value of these findings.

\section{Acknowledgments}

Funding: This study was supported by the National Key Research and Development Program of China (No. 2018YFC1313600), the National Natural Science Foundation of China (No. 82070094), Industry Standard Funding Project of Shanghai Science and Technology (No. 20DZ2202300), the Major Project Matching Funding Project of Shanghai Science and Technology (No. 198012309).

\section{Footnote}

Reporting Checklist: The authors have completed the STROBE reporting checklist. Available at http://dx.doi. org/10.21037/apm-20-1125

Data Sharing Statement: Available at http://dx.doi. org/10.21037/apm-20-1125

Conflicts of Interest: All authors have completed the ICMJE uniform disclosure form (available at http://dx.doi. org/10.21037/apm-20-1125). The authors have no conflicts of interest to declare.

Ethical Statement: The authors are accountable for all aspects of the work in ensuring that questions related to the accuracy or integrity of any part of the work are appropriately investigated and resolved. The study was conducted in accordance with the Declaration of Helsinki (as revised in 2013). The study was approved by ethics committee of Fudan University Affiliated Zhongshan Hospital (No. B2012-073R) and informed consent was taken from all the patients.

Open Access Statement: This is an Open Access article distributed in accordance with the Creative Commons Attribution-NonCommercial-NoDerivs 4.0 International License (CC BY-NC-ND 4.0), which permits the noncommercial replication and distribution of the article with the strict proviso that no changes or edits are made and the original work is properly cited (including links to both the formal publication through the relevant DOI and the license). See: https://creativecommons.org/licenses/by-nc-nd/4.0/.

\section{References}

1. Baguet JP, Barone-Rochette G, Tamisier R, et al. Mechanisms of cardiac dysfunction in obstructive sleep apnea, Nat. Rev. Cardiol 2012;9:679-88.

2. Gottlieb DJ, Yenokyan G, Newman AB, et al. Prospective study of obstructive sleep apnea and incident coronary heart disease and heart failure: the sleep heart health study. Circulation 2010;122:352-60.

3. Parati G, Lombardi C, Narkiewicz K. Sleep apnea: epidemiology, pathophysiology, and relation to cardiovascular risk. Am. J. Physiol. Regul. Integr. Comp. Physiol 2007;293:R1671-83.

4. Dong JY, Zhang YH, Qin LQ. Obstructive sleep apnea and cardiovascular risk: meta-analysis of prospective cohort studies, Atherosclerosis 2013;229:489-95.

5. Celen YT, Peker Y. Cardiovascular consequences of sleep apnea: I-epidemiology. Anadolu Kardiyol Derg 2010;10:75-80.

6. Bodez D, Lang S, Meuleman C, et al. Left ventricular diastolic dysfunction in obstructive sleep apnoea syndrome by an echocardiographic standardized approach:an observational study.Arch Cardiovasc Dis 2015;108:480-90. 
7. Sekizuka H, Osada N, Akashi YJ. Impact of obstructive sleep apnea and hypertension on left ventricular hypertrophy in Japanese patients. Hypertens Res 2017;40:477-82.

8. Baguet JP, Barone Rochette G, Levy P, et al. Left ventricular diastolic dysfunction is linked to severity of obstructive sleep apnoea. Eur Respir J 2010;36:1323-9.

9. Lévy P, Tamisier R, Minville C, et al. Sleep apnoea syndrome in 2011: current concepts and future directions. Eur Respir Rev 2011;20:134-46.

10. Issa O, Peguero JG, Podesta C, et al. Left atrial size and heart failure hospitalization in patients with diastolic dysfunction and preserved ejection fraction. J Cardiovasc Echogr 2017;27:1-6.

11. Tadic M, Cuspidi C, Pencic B, et al. The influence of left ventricular geometry on left atrial phasic function in hypertensive patients. Blood Press 2015;24:361-8.

12. Marin JM, Carrizo SJ, Vicente E, et al. Long-term cardiovascular outcomes in men with obstructive sleep apnoea-hypopnoea with or without treatment with continuous positive airway pressure: an observational study. Lancet 2005;365:1046-53.

13. Kasikcioglu HA, Karasulu L, Durgun E, et al. Aortic elastic properties and left ventricular diastolic dysfunction in patients with obstructive sleep apnea. Heart Vessels 2005;20:239-44.

14. Ring L, Rana BS, Wells FC, et al. Atrial function as a guide to timing of intervention in mitral valve prolapse with mitral regurgitation. JACC Cardiovasc Imaging 2014;7:225-32.

15. Meel R, Khandheria BK, Peters F, et al. Effects of age on left atrial volume and strain parameters using echocardiography in a normal black population. Echo Res Pract 2016;3:115-23.

16. To AC, Flamm SD, Marwick TH, et al. Clinical utility of multimodality LA imaging: assessment of size, function, and structure. JACC Cardiovasc Imaging 2011;4:788-98.

17. Buechel RR, Stephan FP, Sommer G, et al. Head-to-head comparison of two-dimensional and three-dimensional echocardiographic methods for left atrial chamber quantification with magnetic resonance imaging. J Am Soc Echocardiogr 2013;26:428-35.

18. Atas H, Kepez A, Atas DB, et al. Effects of diabetes mellitus on left atrial volume and functions in normotensive patients without symptomatic cardiovascular disease. J Diabetes Complications 2014;28:858-62.

19. Altekin RE, Yanikoglu A, Karakas MS, et al. Assessment of left atrial dysfunction in obstructive sleep apnea patients with the two dimensional speckle-tracking echocardiography. Clin Res Cardiol 2012;101:403-13.

20. Vural MG, Cetin S, Firat H, et al. Impact of continuous positive airway pressure therapy on left atrial function in patients with obstructive sleep apnoea: assessment by conventional and two-dimensional speckle-tracking echocardiography. Acta Cardiol 2014;69:175-84.

21. Çetin S, Vural M, Akdemir R, et al. Left atrial remodelling may predict exercise capacity in obstructive sleep apnoea patients. Acta Cardiol 2018;73:471-8.

22. R.B. Berry, R. Budhiraja, D.J. Gottlieb, et al., Rules for scoring respiratory events in sleep: update of the 2007 AASM manual for the scoring of sleep and associated events. Deliberations of the sleep apnea definitions task force of the American Academy of sleep Medicine. J Clin Sleep Med 2012;8:597-619.

23. Lang RM, Badano LP, Mor-Avi V, et al. Recommendations for cardiac chamber quantification by echocardiography in adults: an update from the American Society of Echocardiography and the European Association of Cardiovascular Imaging. Eur Heart J Cardiovasc Imaging 2015;16:233-70.

24. Anwar AM, Soliman OI, Geleijnse ML, et al. Assessment of left atrial volume and function by realtime three-dimensional echocardiography. Int J Cardiol 2008;123:155-61.

25. Lupu S, Mitre A, Dobreanu D. Left atrium function assessment by echocardiography-physiological and clinical implications. Med Ultrason 2014;16:152-9.

26. Shivalkar B, Van de Heyning C, Kerremans M, et al. Obstructive sleep apnea syndrome: more insights on structural and functional cardiac alterations, and the effects of treatment with continuous positive airway pressure. J Am Coll Cardiol 2006;47:1433-9.

27. Kim SM, Cho KI, Kwon JH, et al. Impact of obstructive sleep apnea on left atrial functional and structural remodeling beyond obesity. J Cardiol 2012;60:475-83.

28. Ho SY, Sanchez-Quintana D, Cabrera JA, et al. Anatomy of the left atrium: implications for radiofrequency ablation of atrial fibrillation. J Cardiovasc Electrophysiol 1999;10:1525-33.

Cite this article as: Wan Q, Xiang G, Xing Y, Hao S, Shu X, Pan C, Li S. Left atrial dysfunction in patients with obstructive sleep apnea: a combined assessment by speckle tracking and real-time three-dimensional echocardiography. Ann Palliat Med 2021;10(3):2668-2678. doi: 10.21037/apm-20-1125 